Provided for non-commercial research and education use. Not for reproduction, distribution or commercial use.

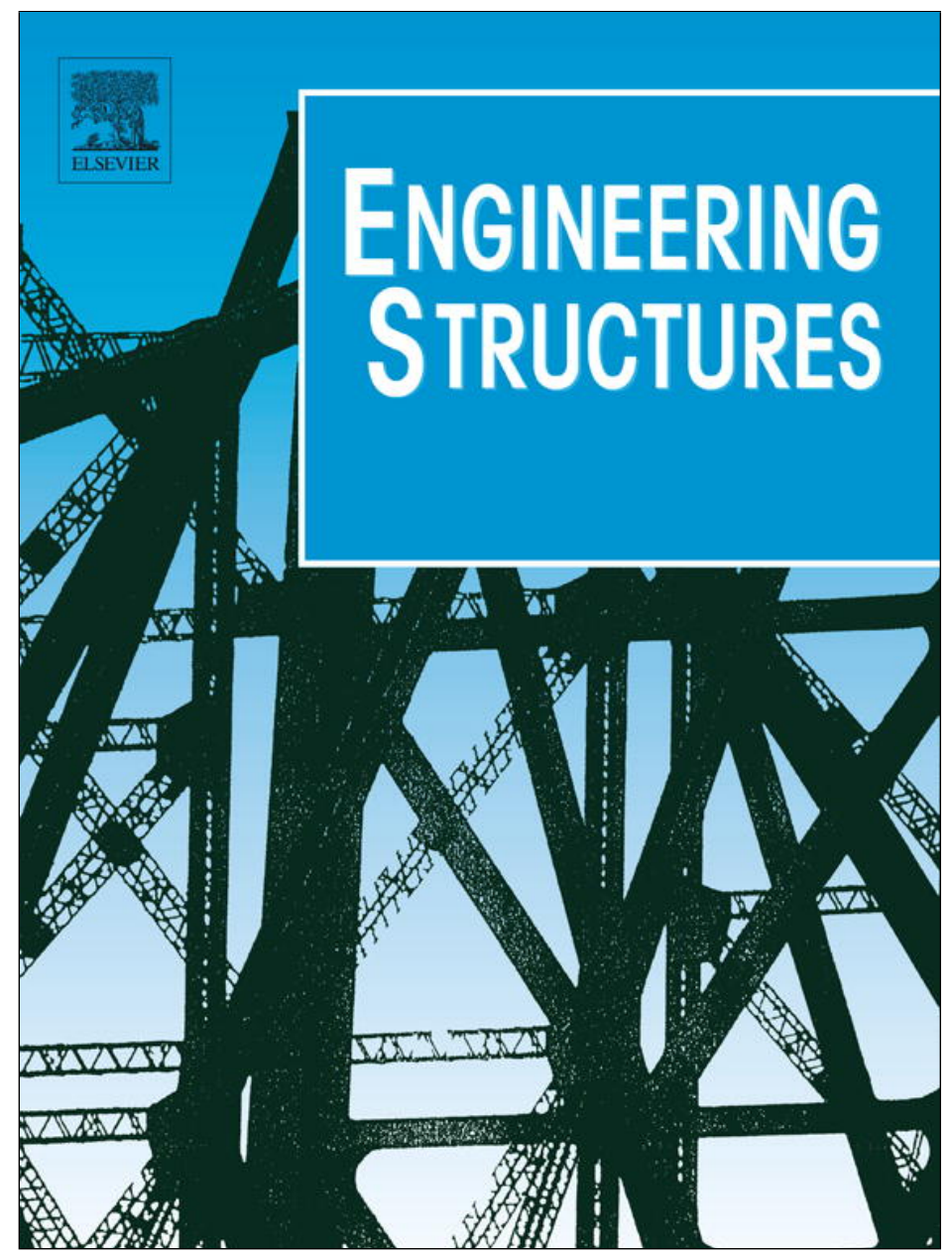

(This is a sample cover image for this issue. The actual cover is not yet available at this time.)

This article appeared in a journal published by Elsevier. The attached copy is furnished to the author for internal non-commercial research and education use, including for instruction at the authors institution and sharing with colleagues.

Other uses, including reproduction and distribution, or selling or licensing copies, or posting to personal, institutional or third party websites are prohibited.

In most cases authors are permitted to post their version of the article (e.g. in Word or Tex form) to their personal website or institutional repository. Authors requiring further information regarding Elsevier's archiving and manuscript policies are encouraged to visit:

http://www.elsevier.com/copyright 


\title{
Experimental and numerical study on structural behavior of a single timber Textile Module
}

\author{
M. Sistaninia, M. Hudert, L. Humbert*, Y. Weinand \\ IBOIS Laboratory for Timber Construction, Ecole Polytechnique Fédérale de Lausanne (EPFL), CH-1015 Lausanne, Switzerland
}

\section{A R T I C L E I N F O}

\section{Article history:}

Received 15 August 2011

Revised 9 August 2012

Accepted 17 August 2012

\section{Keywords:}

Timber Textile Module

Construction stresses

Experimental method

Finite element modeling

\begin{abstract}
A B S T R A C T
The present work investigates an innovative class of timber structure with potential applications in roofing, facade and bridge construction, called Timberfabric. The development of Timberfabric structures originates from the approach of harnessing the structural, modular and qualities of textiles in timber construction (Weinand and Hudert, 2010) [9]. Timberfabric structures are comprised of repetitive arrangements of one or more structural unit cells called Textile Modules. When properly designed, one obtains a modular and lightweight structure with interesting and unusual geometrical and structural qualities.

This paper focuses on the single timber Textile Module. Based on the finite element (FE) method, a reliable procedure is proposed for modeling the overall assembly process of the Textile Module. For comparison, Textile Module prototypes are constructed at two different scales (large and intermediate scales) with different assembly conditions. The proposed geometrically nonlinear FE model allows evaluation of the stresses that are induced during the construction process and which may affect the structural integrity of the module. In particular, the risk of failure during assembly is identified using the anisotropic Tsai-Hill criterion.

The structural behavior of the timber Textile Module is then investigated through bending tests using the constructed prototypes. During the loading procedure, the vertical deflections are measured at different locations on the prototype surface by means of external displacement transducers. Using the FE model, the corresponding deformed shapes are simulated by applying the bending loads on the prestressed Textile Module. Experimental displacements and FE predictions are thus compared and found to be in good agreement.
\end{abstract}

(c) 2012 Elsevier Ltd. All rights reserved.

\section{Introduction}

Wood is a versatile construction material that is abundant in many regions of the world. Moreover, this is a renewable resource that can be processed and assembled in energy efficient ways. Recent studies $[1,2]$ indicate that the use of timber as construction material results in buildings with a better environmental performance in comparison to conventional materials. With regard to present-day concerns over globally increasing energy consumption and simultaneously decreasing resources wood holds a distinct advantage over other construction materials such as concrete or steel. This, in turn, should increase the interest of the research community in expanding the range of applications of timber structures.

Examples of modern but already well-established timber architectural forms include folded plate structures [3,4], lattice

\footnotetext{
* Corresponding author. Tel.: +41216 932398; fax: +41 216932394.

E-mail addresses: masoud.sistaninia@epfl.ch (M. Sistaninia), markus.hudert@epfl.ch (M. Hudert), laurent.humbert@epfl.ch (L. Humbert), yves.weinand@epfl.ch (Y. Weinand).
}

structures (e.g. timber lattice roof for the Mannheim Bundesgartenschau) [4,5] and Multi-reciprocal frame structures [6,7]. Such forms present clear advantages over more traditional flat-surfaced roofing structures, increasing the efficiency of the structure, reducing its weight and enforcing load carrying capacity.

Recently, a new type of timber structures, called Timberfabric, with particular structural properties emanating from the principle of weaving techniques has been developed at IBOIS [8,9]. Its development has been driven by the aim of incorporating specific textile qualities such as modularity and the mutual support of textile fabrics' constituent elements in timber construction. Timberfabric structures have a broad potential for architectural applications due to their versatility, adaptability and their qualities, which are directly linked to their structural make-up. They are based on repetition of a structural unit cell, the Textile Module, which is depicted in Fig. 1a, and which results from bringing together textile assembly principles with timber components. The double-layered Timberfabric structure shown in Fig. 1b represents only one of many possible configurations of Textile Modules. 
(a)

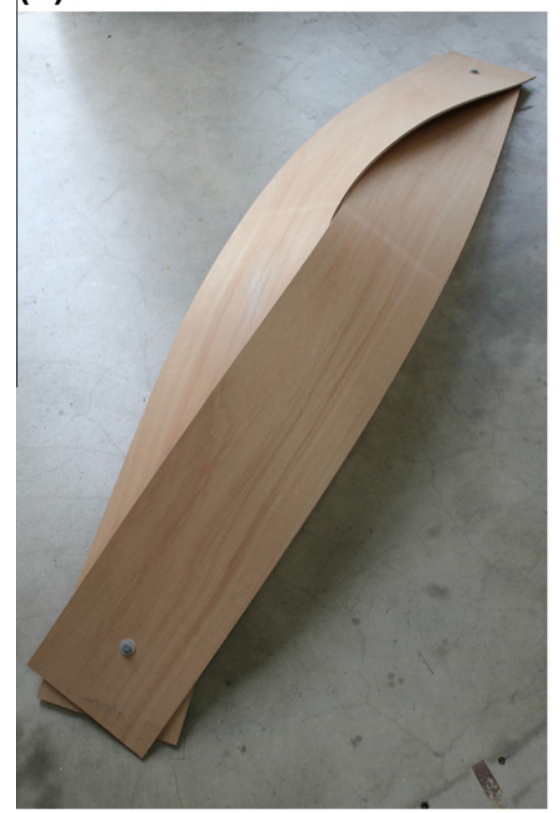

(b)

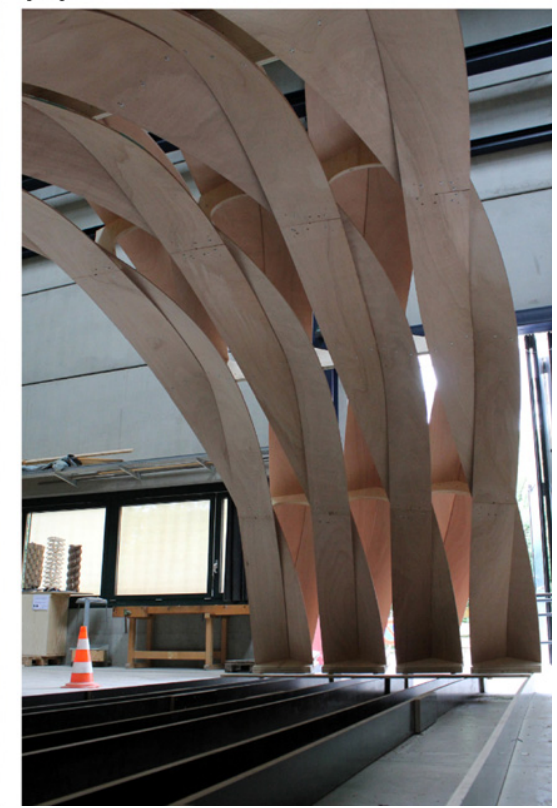

Fig. 1. (a) Single Textile Module. (b) Double-layered Timberfabric structure.

The single Textile Module presented in Fig. 1a provides a structural shape of particular interest for this study. Briefly, it consists of two mutually supporting thin panels that become curved during the assembly process as illustrated in Fig. 2. Consequently, construction (or residual) stresses are generated during the fabrication of the module and their amplitude typically depends on the constitutive material, the size of panels as well as the assembly conditions. The use of poor quality material or inappropriately dimensioned panels or a combination of both may even cause the Textile Module's premature failure during the assembly process. The construction stresses can be evaluated by means of a finite element (FE) model that takes into account the different fabrication stages (Fig. 2).

This paper focuses on the fabrication process and structural behavior of a representative single Textile Module in a bending load configuration. The proposed approach is both experimental and numerical. It involves the fabrication of two prototypes at two different scales (intermediate and large scale) with different assembly conditions, as discussed in the first part of Section 2.
1

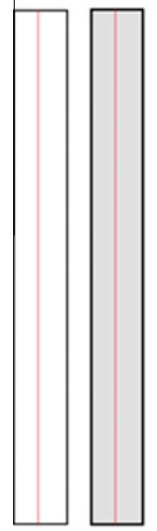

2

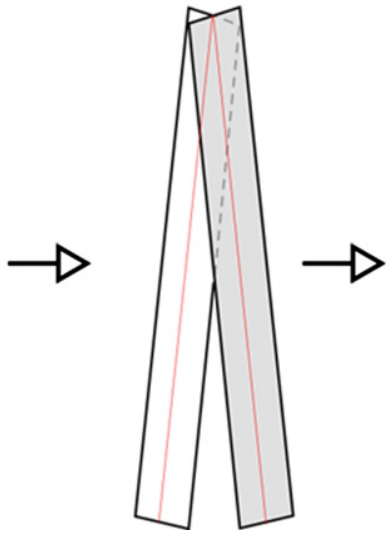

3
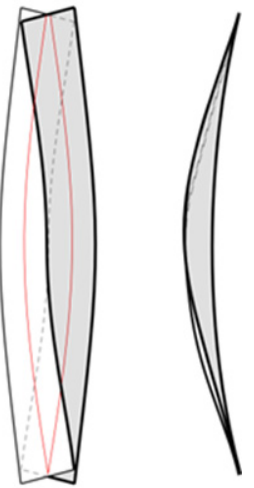

Fig. 2. Design principle for the Textile Module.
The second part of the section is devoted to the bending test setup and required measurement equipment.

Despite the numerous finite element (FE) models already available for braided textile composites [10-14], the numerical study of the timber Textile Module requires special attention as its analysis is complicated by the particular geometry and assembly conditions encountered. Because of the large deflections and rotations undergone by the module during the fabrication stages (Fig. 2), a geometrically non-linear FE model that aims to accurately reproduce the geometrical shape of the Textile Module is developed in Section 3. It is anticipated that this model should permit a representative evaluation of the construction stresses involved in fabrication. In Section 4, vertical displacements measured at several locations of the prototype surface during the bending tests are compared to the finite element predictions. Finally, the structural behavior of the Textile Module is discussed.

\section{Experimental investigations}

\subsection{Materials and specimens}

For this study, two Textile Modules have been constructed: one is formed of two GFP laminated wood panels of length $l=12.320 \mathrm{~m}$ and width $w=0.770 \mathrm{~m}$ and the other is formed of two TeboPly ${ }^{\mathrm{TM}}$ Okoumé plywood panels of $l=2.34 \mathrm{~m}$ and width $w=0.24 \mathrm{~m}$. The GFP and Okoumé panels used to fabricate the Textile Modules are respectively supplied by the companies Schilliger Holz AG (Switzerland) and Thebault (France). As schematically depicted in Fig. 3, the panels consist of symmetric orthotropic laminates, with the three-ply [0/90/0] GFP and four-ply [0/90] $]_{s}$ Okoumé having thickness $t$ of $33 \mathrm{~mm}$ and $6.3 \mathrm{~mm}$ respectively. As customary, plywood is produced from rotary cut veneers that are bonded with an adhesive (synthetic) resin under high pressure conditions. In each case, the uppermost (face) and lowermost (back) veneers are of equal thickness with the same grain direction along the longitudinal axis ( $L$-axis) of the laminate. As depicted in Fig. 3a, for the three-ply configuration, the symmetry plane passes through the center of the core ply of $13 \mathrm{~mm}$ thick and its grain is directed along the transversal axis ( $T$-axis) of the laminate. For the four-ply layup, 

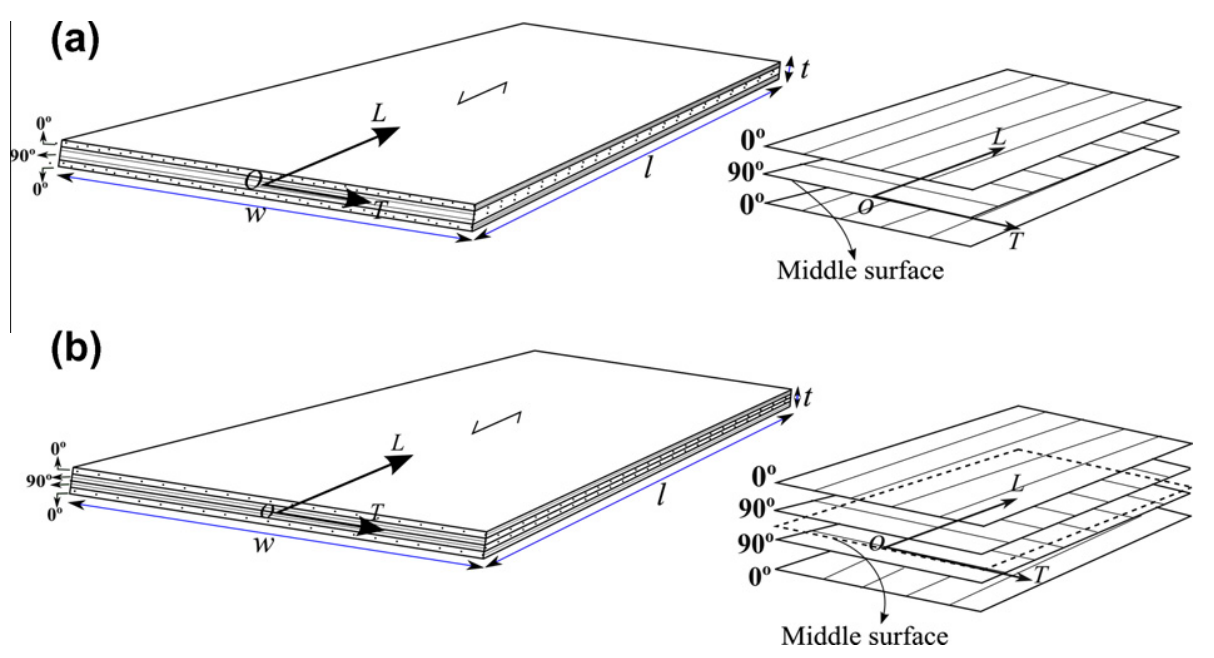

Fig. 3. (a) GFP and (b) Okoumé plywood layups and local coordinate system $(L, T)$.

Table 1

Mechanical properties of GFP and Okoumé laminated wood.

\begin{tabular}{|c|c|c|c|c|c|c|c|c|c|c|c|}
\hline \multirow[t]{3}{*}{ Plywood } & \multicolumn{8}{|c|}{ Characteristic values of strength at fifth percentile (MPa) } & \multirow{2}{*}{\multicolumn{2}{|c|}{$\begin{array}{l}\text { Modulus of elasticity (median values) } \\
\text { Tension and compression (MPa) }\end{array}$}} & \multirow{3}{*}{$\begin{array}{l}\text { Shear modulus (median values) } \\
\text { In plane shear } \\
G_{L T}(\mathrm{MPa})\end{array}$} \\
\hline & \multicolumn{2}{|c|}{ Bending } & \multicolumn{2}{|c|}{ Compression } & \multicolumn{2}{|c|}{ Tension } & \multicolumn{2}{|c|}{ In plane shear } & & & \\
\hline & $f_{m, L}$ & $f_{m, T}$ & $f_{c, L}$ & $f_{c, T}$ & $f_{t, L}$ & $f_{t, T}$ & $f_{v, L}$ & $f_{v, T}$ & $E_{L}$ & $E_{T}$ & \\
\hline $\begin{array}{l}\text { GFP } \\
\text { (3 layers) }\end{array}$ & 15.8 & 2.6 & 7.3 & 4.7 & 4.8 & 3.2 & 1.5 & 1.5 & 6667 & 4333 & 720 \\
\hline $\begin{array}{l}\text { Okoumé } \\
\text { (4 layers) }\end{array}$ & 35 & 32.4 & 10 & 28.5 & 6.1 & 17.4 & 7 & 7 & $\begin{array}{l}2398 \\
4592^{\mathrm{a}}\end{array}$ & $\begin{array}{l}6852 \\
7288^{a}\end{array}$ & 552 \\
\hline
\end{tabular}

${ }^{a}$ Averaged values obtained in the laboratory.

the two core plies are of the same thickness $(2 \mathrm{~mm})$ and glued to each other so that their grain direction remains perpendicular to the longitudinal axes of the face veneers (along the $T$-axis). This glued interface corresponds to the symmetry plane (middle surface) of the plywood. Such an even-layered arrangement has proven to increase efficiency in veneer manufacturing and grading (less variability) although adding to the cost of production.

Both the GFP and Okoumé panels are composed of orthotropic layers (veneers) having principal material axes that coincide with the longitudinal $(L)$ and transversal $(T)$ geometrical directions of the panels (see Fig. 3). On a macroscopic scale, they can be treated as homogeneous orthotropic materials with the $L$ and $T$ axes as the principal axes of the equivalent material.

Homogenized elastic material properties, provided by the manufacturers at the standard conditions (temperature of $20^{\circ} \mathrm{C}$ and a relative humidity of $65 \%$ ), are reported in Table 1 , where the $L$ and $T$ subscripts refer to the longitudinal and transversal directions, respectively. Also provided in Table 1 are the longitudinal and transversal moduli of the Okoumé panels measured in the environmental conditions of the laboratory. Tensile tests were carried out in a standard crosshead testing machine (in displacement control with a crosshead speed of $2 \mathrm{~mm} / \mathrm{min}$ ) approximately eight months after receiving the panels from the manufacturer. The averaged values of the elastic moduli reported in Table 1 are calculated on the basis of measurements obtained using four samples with the direction of the grain of the face layers parallel to the loading direction and two samples with the direction of the grain of the face layers perpendicular to the loading direction. These values will subsequently be used in the finite element simulations.

For simplicity, hereafter the GFP Textile Module will be referred to as the large-scale (TM1) specimen and the Okoumé Textile Module as the intermediate-scale (TM2) specimen. Besides their differences in size and material properties, the modules are assembled in a different way. For prototype TM1, the ends of the panels are mechanically linked by means of a pinned connection while hardwood wedge connectors that restrain all degree of freedom are employed to build the second prototype TM2. The construction process of the two prototypes is described in detail below.

\subsection{Construction of the Textile Modules}

\subsubsection{Pin connection}

A pinned connection is used for the assembly of Textile Module TM1. Practically, the two panels are connected to each other at their ends using a system of steel threaded rods with nuts and washers. The starting point for the assembly procedure is illustrated in Fig. 4. For convenience, the global reference coordinate system $(x, y, z)$ is introduced such that the vertical $z$-axis crosses panel 1 and panel 2 at points A1 and A2, respectively. These two points have the same geometrical position, taken as the origin $\mathrm{A}$ of the global system. Strictly speaking, point A1 belongs to the lower face of panel 1 while point A2 is on the upper face of panel 2 , with the same in-plane coordinates $L=w / 2, T=0$ in the local frame $(L, T)$ of the panels (see Fig. 3 ). The middle surfaces of the panels are positioned parallel to the plane $(x, y)$ and symmetrically rotated about the $z$-axis by an angle $\alpha=10^{\circ}$. Then, the panels are linked to each other at points A1 and A2 using the steel threaded rod (not represented in Fig. 4), thus restraining their relative displacement at A while keeping the rotation free along the threaded rod axis. Note that the threaded rod axis initially coincides with the $z$-axis but does not keep this direction after assembly.

Let us now consider the opposite points C1 and C2 (see Fig. 4) having local in-plane coordinates $(L=l-w / 2, T=0)$ such as $\mathrm{AC} 1=\mathrm{AC2}=l-w=11.55 \mathrm{~m}$. The line $(\mathrm{C} 1 \mathrm{C} 2)$ lies perpendicular to the $y$-axis, bisecting it at point $C^{\prime}$. In other words, one can trace an isosceles triangle (AC1C2) whose base is (C1C2). Practically, 


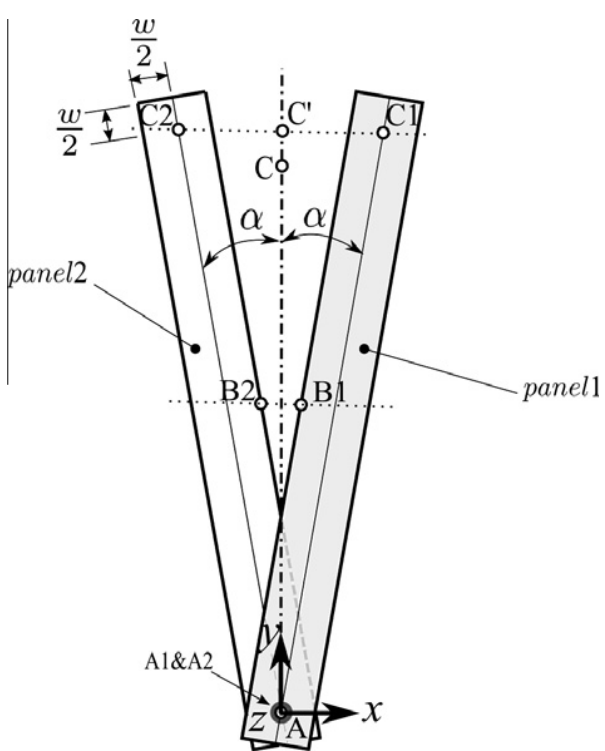

Fig. 4. Assembly procedure and associated coordinate system.

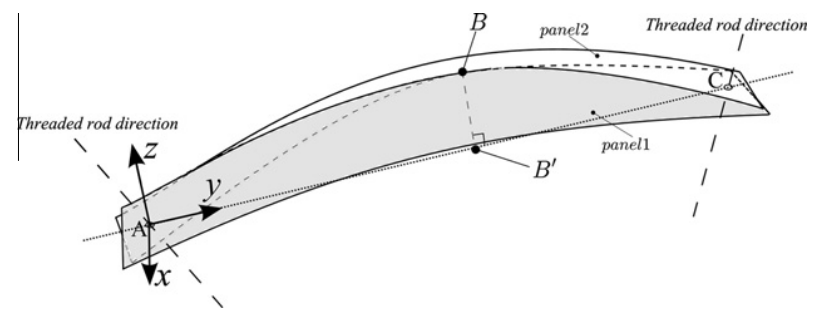

Fig. 5. Textile Module geometry with curved panels.

the panels are pulled together at points $\mathrm{C} 1$ and $\mathrm{C} 2$ by means of cables to a third common point $C$ lying on the $y$-axis. The distance $A C$ is typically less than $A C^{\prime}$. The panels that become curved are again pin-connected at $C$ by means of a second threaded rod. During this operation, the mid-points B1 and B2 of two panel edges come into contact at a point $\mathrm{B}$ that corresponds to the maximum height of the resulting structure (Fig. 5). The maximum height (i.e. distance $\mathrm{BB}^{\prime}$ ) and span (i.e. distance $\mathrm{AC}$ ) for TM1, are respectively $1.5 \mathrm{~m}$ and $10.05 \mathrm{~m}$.

It is worth noting that the two threaded rod axes and point $B$ belong to the vertical plane $(y, z)$. Denoting $\mathrm{B}^{\prime}$ the vertical projection of $B$ onto the $y$-axis, one has an isosceles triangle ABC of height equal to the line segment $\mathrm{BB}^{\prime}$ and base $\mathrm{AC}=2 \mathrm{AB}^{\prime}$. Moreover, the two threaded rod axes are symmetrically oriented with respect to the axis $\mathrm{BB}^{\prime}$.

The two ends of the Textile Module TM1 are firmly fixed on the ground by using a special steel connector (see Fig. 6a), restraining thus all displacements and rotations along the corresponding panel edges.

Various assembly conditions can readily be imposed on the panels of Textile Module, modifying accordingly its final shape and structural behavior. The selection of the type of connectors to be used depends on the assembly conditions under consideration.

Finally, it should be noted that apparently minor alterations to the assembly conditions may have a significant influence on the strength of the structure. An early prototype (TM0) was composed of panels whose characteristics were similar to those of TM1 except that the width $w$ was equal to $1.650 \mathrm{~m}$. This prototype was broken in two after the nuts were tightened in the final step. Both panels failed in two parts at the middle (Fig. 6b) because of the stresses induced during assembly.

\subsubsection{Wedge connection}

A second type of connection, referred simply to as "wedge connection", is employed for the construction of prototype TM2. As indicated in Fig. 7, two identical sets of three wedge elements are used to connect the panels to each other and to simultaneously fix the whole structure on the ground. The different elements are machined by cutting a block of hardwood of length $20 \mathrm{~cm}$ and cross-sectional area $w / 2 \times w / 4=12 \times 6 \mathrm{~cm}^{2}$ along two oblique cutting planes with different orientations (see Fig. 7). The resulting uppermost (Wedge 3) and lowermost (Wedge 1) elements thus have one cross section at right angle and the other obliquely oriented with respect to the lateral faces. Before cutting, three holes of diameter $10 \mathrm{~mm}$ have been drilled along the length of the wooden block as indicated in Fig. 7, for the introduction of a tightening system composed of threaded rods and nuts.

Positioning the structure on the ground is achieved by means of the supporting element Wedge 1 . The intermediate element (Wedge 2 ) permits the relative position of the panels to be accurately controlled on the constrained areas (assembly conditions). The third wedge element (Wedge 3 ) is practically used to connect the panels together by simply tightening the nuts, thus aligning the three wedges vertically. Moreover, this locking device allows the module to be fully clamped on the ground.The positions of the two oblique (cross-sectional) planes can readily be described by means of six assembly parameters $\left(\theta_{x 1}, \theta_{x 2}, \theta_{y 1}, \theta_{y 2}, d, d^{\prime}\right)$ as presented in Fig. 8. The origin $\mathrm{O}$ of the reference coordinate system $(x, y, z)$ is (a)

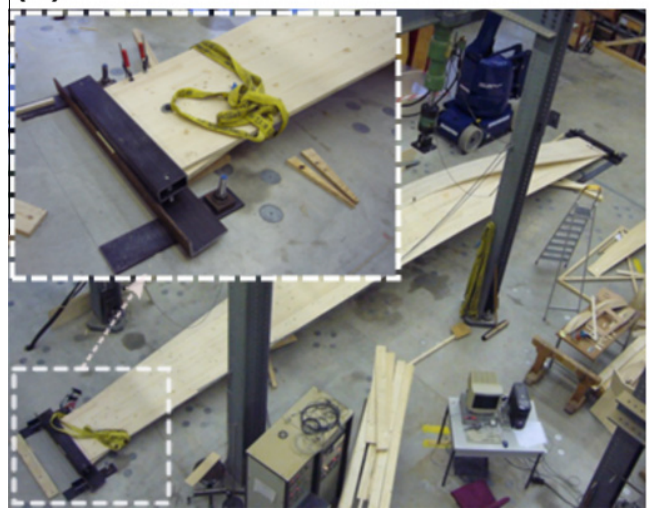

(b)

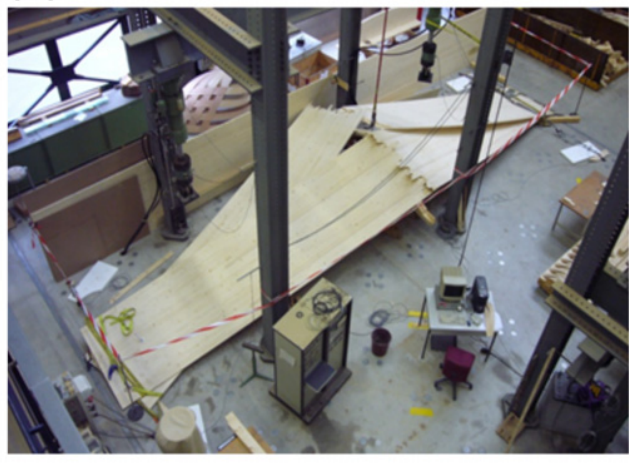

Fig. 6. (a) Prototype TM1 and steel connector used to fix it on the floor. (b) Failure during the assembly process. 


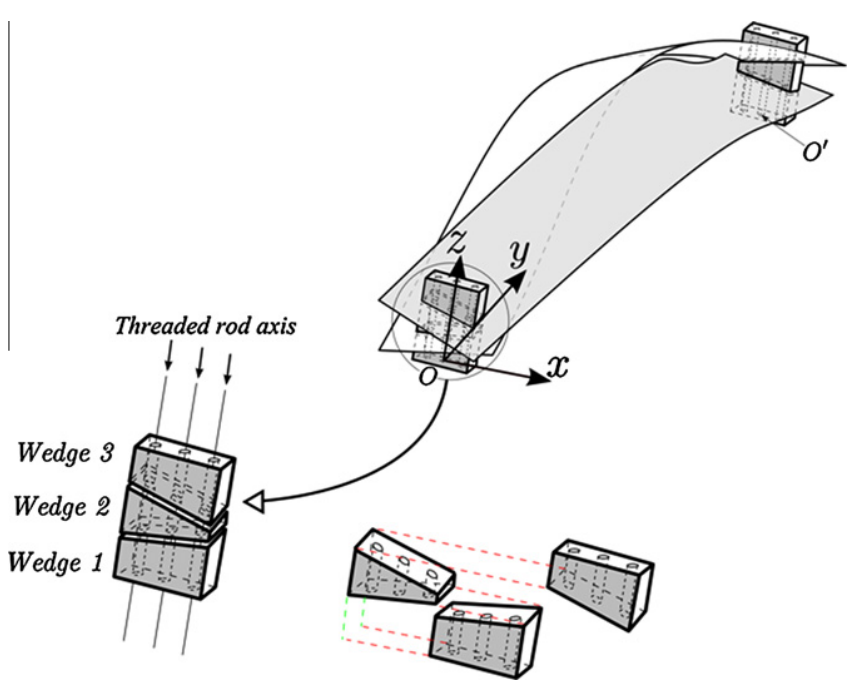

Fig. 7. Textile Module assembled with wedge connection.

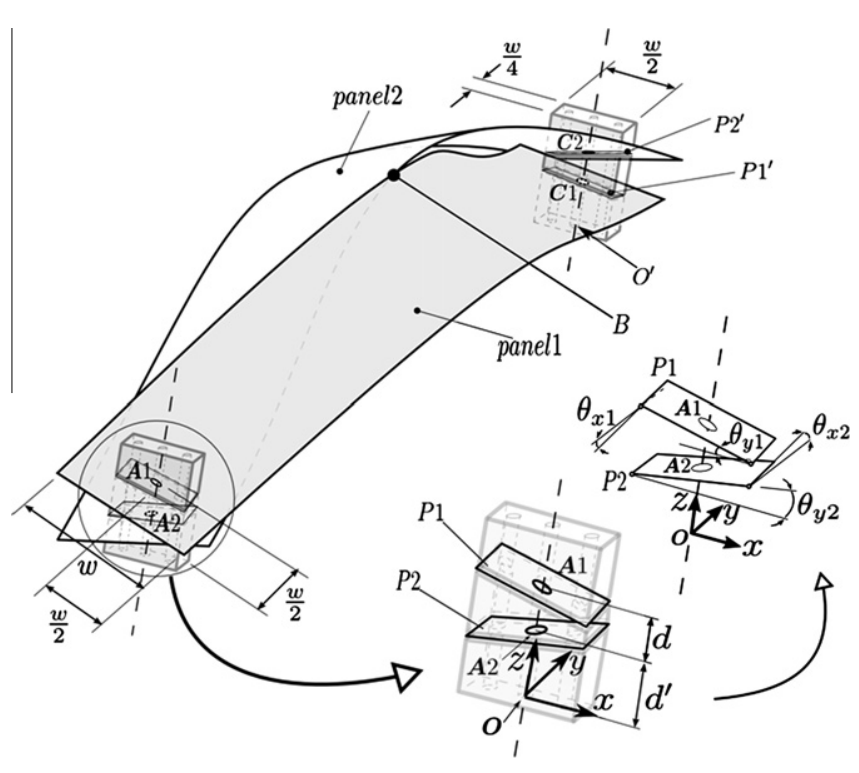

Fig. 8. Definition of the assembly parameters.

placed at the center of the bottom section of Wedge 1 . Sketched in Fig. 8 is the final shape of the module where the vertical $z$-axis crosses the panel 1 and panel 2 at points $A 1$ and $A 2$ whose respective coordinates are $\left(0,0, d+d^{\prime}\right)$ and $\left(0,0, d^{\prime}\right)$. Unlike the situation for prototype TM1, these two points do not occupy an identical position because of the introduction of Wedge 2 . It must also be noted that the threaded rod axes, keep a fixed orientation corresponding to the vertical $z$-axis during the assembly. Moreover, A1 and A2 are centroidal points for the contact areas $p 1$ and $p 2$, whose orientations with respect to the $x$-axis and $y$-axis are given by the two angles $\theta_{x 1}, \theta_{y 1}$ and $\theta_{x 2}, \theta_{y 2}$ respectively.

Similarly, the opposite points $\mathrm{C} 1$ and $\mathrm{C} 2$ are centroidal points for the contact areas $p 1^{\prime}$ and $p 2^{\prime}$ as defined in Fig. 8. Point $\mathrm{C} 1$ is now located under point $\mathrm{C} 2$ and their respective coordinates are given by $\left(0, s, d^{\prime}\right)$ and $\left(0, s, d+d^{\prime}\right)$. Denoting $\mathrm{O}^{\prime}$ the intersection of the vertical axis passing through these points and the $y$-axis, one obtains $s=\mathrm{OO}^{\prime}$. In this case, the angles $-\theta_{x 1}$ and $-\theta_{y 1}$ (resp. the angles $-\theta_{x 2}$ and $-\theta_{y 2}$ ) orientate the plane $p 2^{\prime}$ (resp. the plane $p 1^{\prime}$ ) with respect to the $x$-axis and $y$-axis.

As was the case for prototype TM1, an isosceles triangle $\mathrm{OBO}^{\prime}$ can be constructed where vertex $\mathrm{B}$ is defined as the common position of the contacting points B1 and B2. Again, $\mathrm{B}^{\prime}$ denotes the vertical projection of the vertex $\mathrm{B}$ on the opposite side $\mathrm{OO}^{\prime}$.

Fig. 9 shows a TM2 module that is fixed on a thick wooden base using the previously described wedge connections. The maximum height $\mathrm{BB}^{\prime}$ corresponds to $0.360 \mathrm{~m}$, the span $s=2.056 \mathrm{~m}$ and the assembly parameters are chosen to be $\theta_{x 1}=17^{\circ}, \theta_{x 2}=11.5^{\circ}, \theta_{y 1}$ $=14.5^{\circ}, \theta_{y 2}=-8^{\circ}, d^{\prime}=76 \mathrm{~mm}, d=47.7 \mathrm{~mm}$.

\subsection{Test setups and measurement equipment}

The test setups used for the experiments carried out on prototypes TM1 and TM2 is shown in Fig. 10a and b, respectively.

The ends of prototype TM1 are clamped on the floor by means of the steel connector shown in Fig. 6. A hydraulic actuator from Walter\&Bai Company with a maximum force capacity of $\pm 500 \mathrm{kN}$ is used to load the prototype at mid span. An additional V-shaped element (made of hardwood) is attached to the actuator for transferring the load on each panel as indicated in Fig. 10a and Fig. 11a. During the test, the load is applied up to the ultimate limit state under displacement control with a constant displacement rate of $6 \mathrm{~mm} / \mathrm{min}$.

Prototype TM2 and its wooden base are placed on two concrete blocks as shown in Fig. 7. In this case, a hydraulic actuator with a maximum force capacity of $\pm 300 \mathrm{kN}$ is used for applying the bending load at the prototype mid span. For an accurate measurement of the force, a load cell of $5 \mathrm{kN}$ is added as indicated in the insert of Fig. 10. A specially-designed wooden element with two steel parallel rods is fastened to the actuator for distributing the load on each panel (see Fig. 10b and Fig. 11b). Again, the test is run under displacement control (constant rate of $3 \mathrm{~mm} / \mathrm{min}$ ) and the prototype loaded up to $0.5 \mathrm{kN}$.

In both cases, the resulting deflections are recorded at several positions along the prototype surface using external transducers

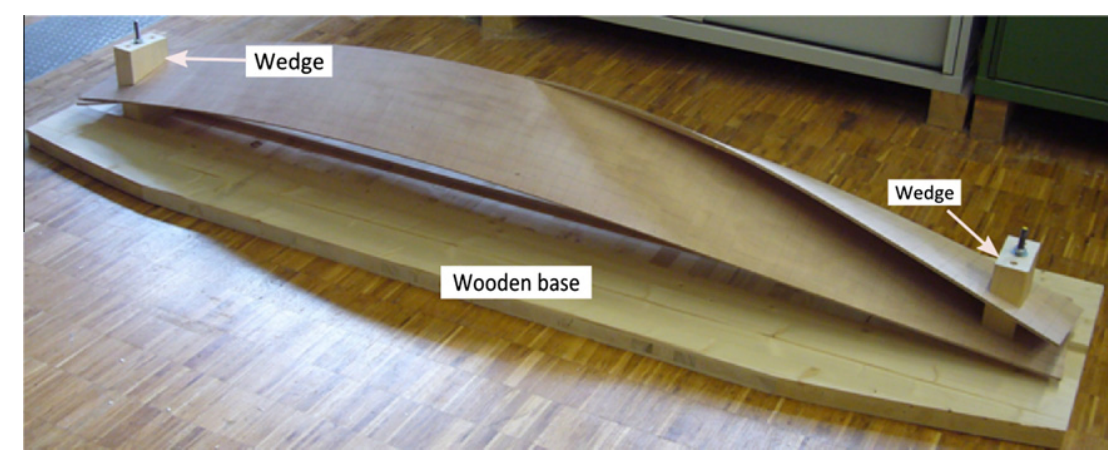

Fig. 9. Textile Module TM2 with wooden base. 
(a)
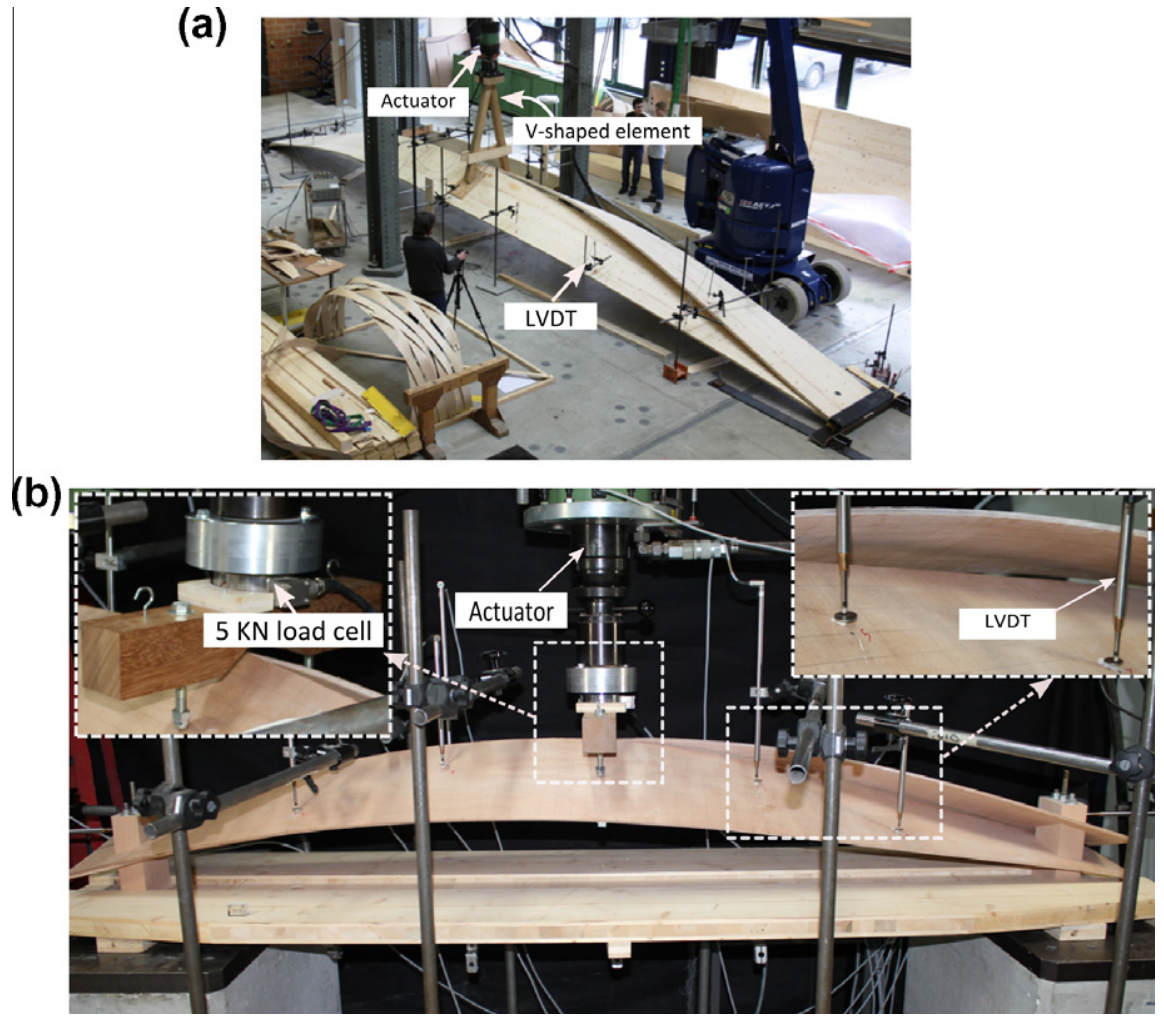

Fig. 10. Test setup for (a) prototype TM1 and (b) prototype TM2.
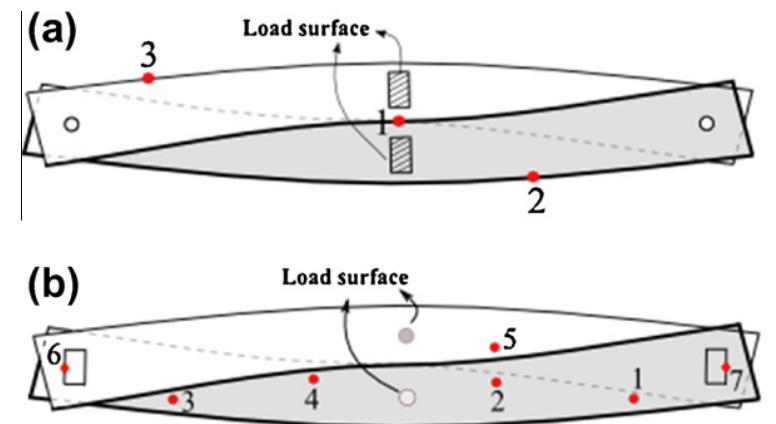

Fig. 11. Location of measurement points for (a) TM1 and (b) TM2 (top view).

mounted on self-supporting vertical steel rods as shown in Fig. 10a and Fig. 10b. Specifically, linear variable differential transducers (LVDTs) whose measurement range is $\pm 100 \mathrm{~mm}$ are employed for measuring the vertical component of the displacement for prototype TM1. Data are typically recorded at three positions (numbered as 1, 2 and 3 in Fig. 11a) and the displacement of the actuator is measured by using a LVDT mounted on the actuator. The positions of LVDTs in the local frame $(L, T)$ are: LVDT1 $(L=6.1875 \mathrm{~m}, T=-0.4125 \mathrm{~m})$, LVDT2 $(L=8.075 \mathrm{~m}, T=0.4125 \mathrm{~m})$ and LVDT3 $(L=1.25 \mathrm{~m}, T=0.4125 \mathrm{~m})$.

For prototype TM2, LVDTs with measurement ranges of $\pm 30 \mathrm{~mm}$ and $\pm 50 \mathrm{~mm}$ are placed at the seven positions indicated in Fig. $11 \mathrm{~b}$. Those with measurement ranges of $\pm 50 \mathrm{~mm}$ are only used to record the vertical displacements at points 4 and 5. For positions 6 and 7 , the transducers are directed perpendicular to the top wedge connection in order to check its deformation along the horizontal direction ( $y$-axis) during loading. As can be observed in the enlarged region of Fig. 10b, small circular pieces of metal are glued on the panels to provide a flat mounting surface in order to maintain the contact with the transducers during the test. A grid mesh composed of $78 \times 8$ elements of $3 \times 3 \mathrm{~cm}^{2}$ size (not visible in the images presented), is also drawn on the upper surface of each panel. This grid corresponds to the numerical mesh used in the finite element analysis. Finally, the positions of LVDTs in the local frame $(L, T)$ are given by: LVDT1 $(L=0.54 \mathrm{~m}, T=0.06 \mathrm{~m})$, LVDT2 ( $L=$ $0.84 \mathrm{~m}, T=-0.03 \mathrm{~m})$, LVDT3 $(L=1.8 \mathrm{~m}, T=0.06 \mathrm{~m})$, LVDT4 $(L=$ $1.5 \mathrm{~m}, T=-0.03 \mathrm{~m})$ and LVDT5 $(L=0.84 \mathrm{~m}, T=0.03 \mathrm{~m})$.

\section{Numerical modeling}

A 3D geometrically nonlinear finite element analysis is proposed in this part to simulate the assembly process of the Textile Module, including fixation of the pinned and wedge connections. It is aimed to calculate the construction stresses (initial stresses) and to reproduce the structural behavior of the structure under bending static loads.

\subsection{Material properties}

In this study, GFP and Okoumé plywood are modeled as singlelayer orthotropic linear elastic materials assuming that the layers are rigidly bonded together. When these simplifying assumptions are taken into account, only four independent engineering constants are required to fully characterize the material behavior, namely: the longitudinal $E_{L}$ and transversal $E_{T}$ Young's moduli, in-plane shear modulus $G_{L T}$ and Poisson's ratio $v_{L T}[15,16]$. The equivalent unidirectional lamina is consequently assumed to be under a state of plane stress with principal material directions ( $L$, $T$ ) indicated in Fig. 3. The equivalent material properties given in Table 1 are used for the numerical analysis. In the calculation, the average value $v_{L T}=0.3$ will be considered for the Poisson's 
ratio. Volumetric changes (shrinkage or swelling of wood panels) due to moisture loss or gain are not taken into account here.

\subsection{Finite element model}

The commercial FE code ABAQUS is used to build a 3D model of the assembly process for the Textile Module. Because large rotations and deflections as well as boundary nonlinearities (i.e. sudden change in contact conditions between the panels) are expected, a geometrically nonlinear analysis is considered where the specified displacements and loads are applied incrementally.

Each panel is discretized by means of six-node triangular thin shell elements of uniform size (no refinement). Specifically, fully integrated STRI65 elements with five degrees of freedom per node (three displacements $u_{x}, u_{y}, u_{z}$ and two rotations $\theta_{x}, \theta_{y}$ ) are used. These elements, adapted for large rotation but small strain, provide accurate solutions in the framework of the classical (Kirchhoff) shell theory, where the shell normal remains perpendicular to the shell reference surface (i.e. negligible transverse shear) [1719].

An implicit time-integration procedure that relies on the Newton-Raphson iterative scheme [20] is typically used to solve the simultaneous incremental nonlinear equations. Accordingly, an estimate of the incremental displacement field satisfying the displacement and traction boundary conditions is obtained at the end of a generic time increment. In this analysis, the calculations are achieved with a maximum time step of 0.1 for each increment.

\subsection{Boundary and assembly conditions}

The simulation of the Textile Module with pinned and wedge assembly conditions requires considering the construction procedure presented in Section 2.2. The same nomenclature is repeated here. Thus, rectangular shell surfaces are used to represent the panels and are positioned in the global coordinate system $(x, y, z)$ of Abaqus as indicated in Section 2.2. In particular, they are symmetrically rotated by an angle $\alpha\left(\alpha=10^{\circ}\right.$ is taken here) about the global $z$-axis. The assembly process is then simulated by applying appropriate boundary and assembly conditions on the panels as detailed below for the pinned and wedge connections.

For the pinned connection (prototype TM1), the modeling procedure is as follows:

- The boundary conditions $u_{x}=u_{y}=u_{z}=0\left(u_{i}\right.$ is the displacement component in the $i$-axis direction) are imposed at points $\mathrm{A} 1$ (belonging to panel 1) and A2 (belonging to panel 2). Recall that these points are located at the origin $A$ of the frame.

- At points C1 and C2 (Fig. 4), only the vertical displacement $u_{z}=0$ is imposed. Consequently, these points are constrained to stay in the plane $(x, y)$.

- The displacement vectors $\mathbf{u}_{\mathrm{C} 1}=-u_{\mathrm{C}} \hat{\mathbf{e}}_{1}$ and $\mathbf{u}_{\mathrm{C} 2}=u_{\mathrm{C}} \hat{\mathbf{e}}_{1}\left(\hat{\mathbf{e}}_{1}\right.$ is the unit vector in $x$ direction) are applied to points $\mathrm{C} 1$ and $\mathrm{C} 2$ respectively to match them to the common point $C^{\prime}$ of the $y$-axis such as

$u_{C}=(l-w) \sin (\alpha)$

where $u_{C}$ is equal to $2005.64 \mathrm{~mm}$ and $364.66 \mathrm{~mm}$ for TM1 and TM2, respectively.

- The mid-points B1 and B2 of the panel edges are simultaneously shifted to the contact point $\mathrm{B}$, by applying the displacement vectors $\mathbf{u}_{\mathrm{B} 1}=-u_{B} \hat{\mathbf{e}}_{1}+w_{B} \hat{\mathbf{e}}_{3}$ and $\mathbf{u}_{\mathrm{B} 2}=u_{B} \hat{\mathbf{e}}_{1}+w_{B} \hat{\mathbf{e}}_{3}\left(\hat{\mathbf{e}}_{3}\right.$ is the unit vector in $z$ direction) at points $\mathrm{B} 1$ and $\mathrm{B} 2$ respectively. The expression of $u_{B}$ for this transformation can be determined as

$u_{B}=(l-w) / 2 \cdot \sin (\alpha)-w / 2 \cdot \cos (\alpha)$ with respective numerical values of $623.66 \mathrm{~mm}$ and $64.15 \mathrm{~mm}$ for TM1 and TM2.

The parameter $w_{B}$ measured by the experimental prototype is imposed, modifying the $y$-coordinate of point $C^{\prime}$ that becomes point $C$ (see Fig. 4 and Fig. 5 ). Note that $w_{B}$ corresponds to the distance $\mathrm{BB}^{\prime}$ in Fig. 5 .

For the wedge connection (prototype TM2), the modeling procedure is as follows:

- The boundary conditions $u_{x}=u_{y}=0$ are imposed at points A1 and $\mathrm{A} 2$.

- $u_{z}=d^{\prime}$ is imposed at points A2 and C1, while $u_{z}=d^{\prime}+d$ is imposed at points A1 and C2. For the definition of $d$ and $d^{\prime}$ see Fig. 8.

- $\theta_{x}= \pm \theta_{x 1}, \theta_{y}= \pm \theta_{y 1}$ are applied to constrain the nodes on the areas $p 1$ and $p 2^{\prime}$ and $\theta_{x}= \pm \theta_{x 2}, \theta_{y}= \pm \theta_{y 2}$ to constrain the areas $p 2$ and $p 1^{\prime}$

- The displacements in $x$-direction are obtained at points C1 and C2 using the displacement vectors $\mathbf{u}_{\mathrm{C} 1}=-u_{\mathrm{C}} \hat{\mathbf{e}}_{1}, \mathbf{u}_{\mathrm{C} 2}=u_{\mathrm{C}} \hat{\mathbf{e}}_{1}$ and Eq. (1). As previously, the points are free to move in the $y$-direction to the common point $\mathrm{C}$ such as $\mathrm{AC}=s$.

- For the wedge connection, only one displacement component (in $x$ direction) is needed to position points B1 and B2 to the common point $\mathrm{B}$. So, the displacement vectors $\mathbf{u}_{\mathrm{B} 1}=-u_{B} \hat{\mathbf{e}}_{\mathbf{1}}$ and $\mathbf{u}_{\mathrm{B} 2}=u_{\mathrm{B}} \hat{\mathbf{e}}_{1}$ should be applied to points B1 and B2 respectively. The expression of $u_{B}$ is given by Eq. (2).

\subsection{Contact interaction}

To prevent interpenetration of the two panels, contact regions have to be defined. The general finite-sliding, surface-to-surface algorithm of Abaqus/Standard is used, allowing arbitrary large sliding as well as large rotations and deformations of the surfaces. A frictionless hard contact pressure-overclosure relationship is considered, for which the penetration of the slave surface into the master surface is minimized and no tensile stress is transferred trough the interface. Master (MS1, MS2) and slave (SS1, SS2) surfaces that can potentially come into contact are specified in Fig. 12 by dividing each panel in two regions. Moreover, surface SS1 (resp. SS2) is located on the lower face of panel1 (resp. panel 2) while MS1 (resp. MS2) belongs to the upper face of panel1 (resp. panel 2). The two contact surface pairings (MS1, SS2) and (MS2, SS1) can then be identified for the tracking contact algorithm.

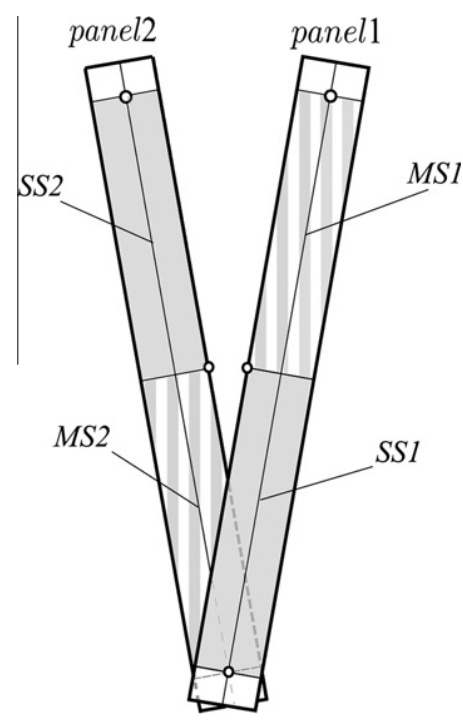

Fig. 12. Definition of contact interaction between the panels. 
(a)

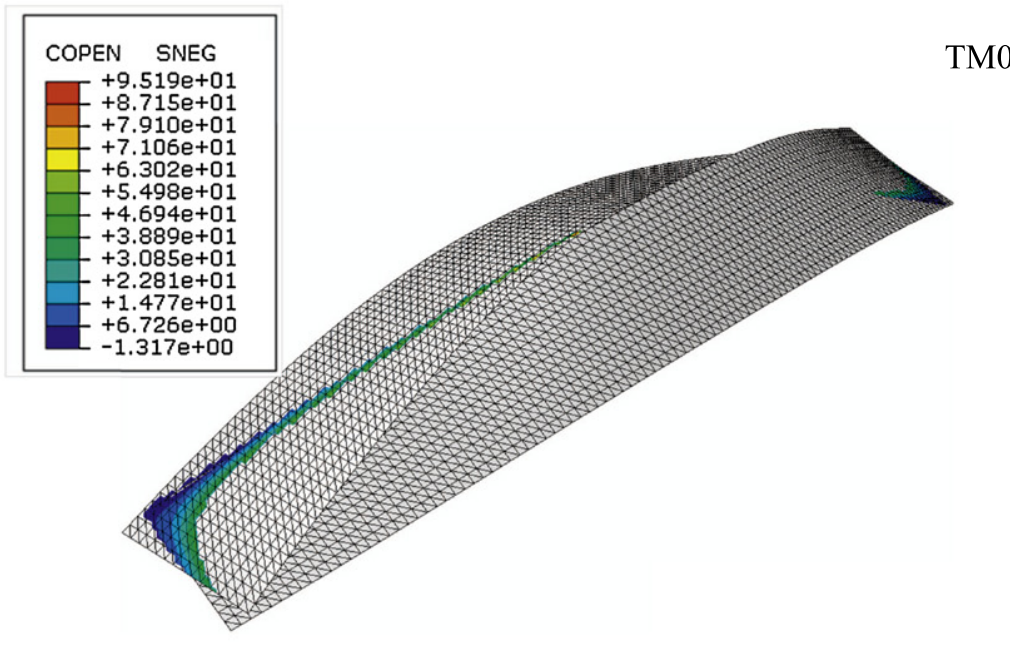

TM1
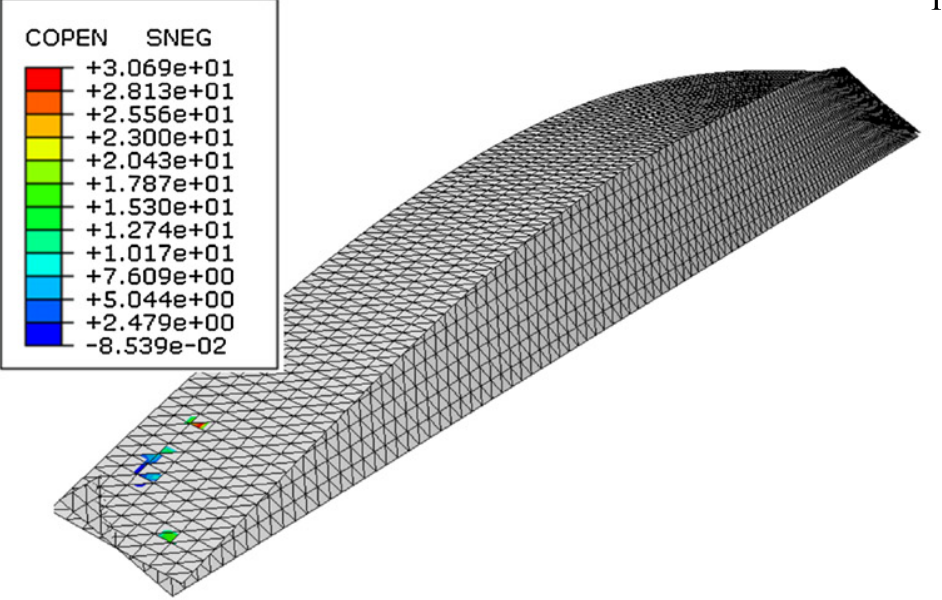

TM2
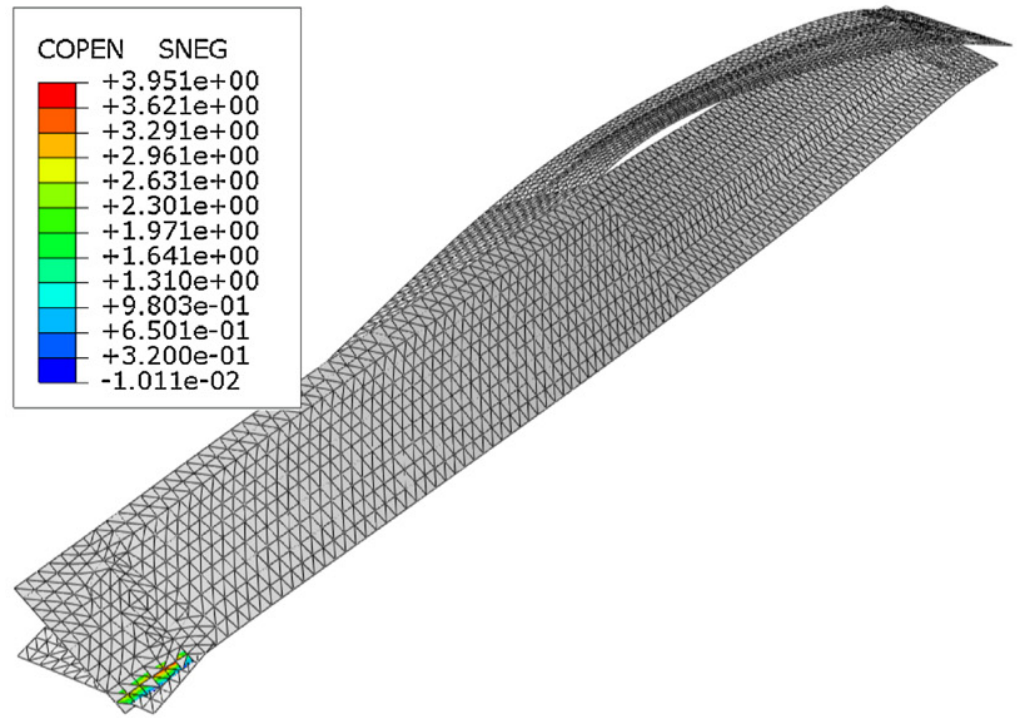

(b)
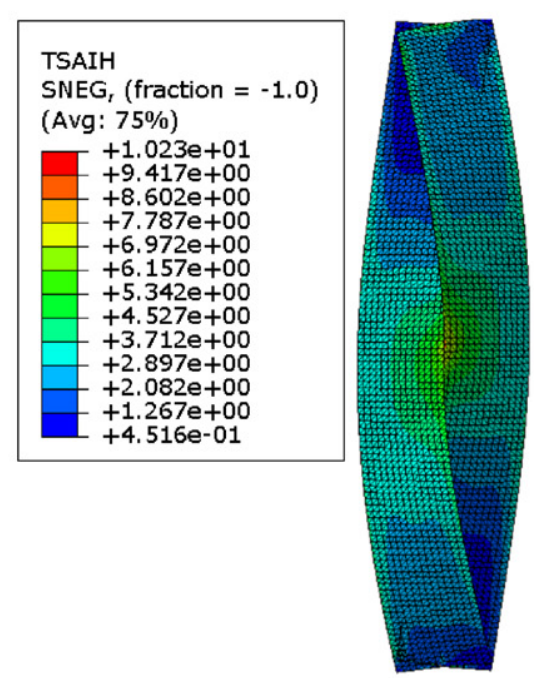

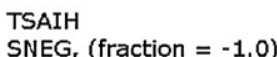

(Avg: 75\%)

\begin{tabular}{|l}
\hline \\
$+1.350 \mathrm{e}+00$ \\
$+1.246 \mathrm{e}+00$ \\
$+1.142 \mathrm{e}+00$ \\
$+1.038 \mathrm{e}+00$ \\
$+9.341 \mathrm{e}-01$ \\
$+8.301 \mathrm{e}-01$ \\
$+7.261 \mathrm{e}-01$ \\
$+6.221 \mathrm{e}-01$ \\
$+5.181 \mathrm{e}-01$ \\
$+4.142 \mathrm{e}-01$ \\
$+3.102 \mathrm{e}-01$ \\
$+2.062 \mathrm{e}-01$ \\
$+1.022 \mathrm{e}-01$ \\
\hline
\end{tabular}

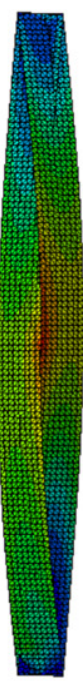

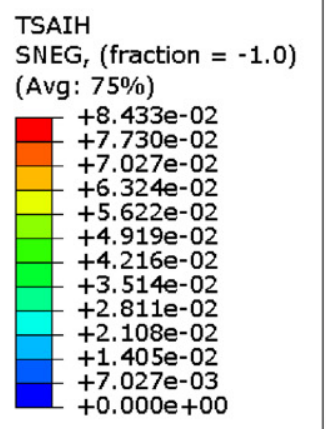

Fig. 13. (a) Simulated initial shapes and visualization of clearance values between contacting surfaces of TM0, TM1 and TM2 in mm. (b) Representation of the Tsai-Hill criterion $I_{F}$. 
Frictional effects can be included by considering the (simplest) classical isotropic Coulomb model to describe the frictional behavior between the contacting panels. Representative values of 0.3 and 0.5 will be taken for the (static) coefficient of friction $\mu$ in our case.

\subsection{Numerical results}

Fig. 13a shows the 3D initial shapes obtained numerically for the large-scale and intermediate-scale Textile Modules. In each case, the contact opening variable COPEN (in $\mathrm{mm}$ ) corresponds to the clearance between the potentially contacting surfaces. Negative values of COPEN in Fig. 13a indicate small overclosures of the surfaces. For the large scale geometry, two widths have been considered for the panels (i.e. $w=1.650 \mathrm{~m}$ and $w=0.77 \mathrm{~m}$ ), corresponding to the prototypes TM0 and TM1. The FE mesh incorporates STRI65 shell elements, with an element mesh size of $110 \mathrm{~mm}$. Preliminary calculations were conducted with several element mesh sizes ranging from 80 to $150 \mathrm{~mm}$, revealing a difference less than $2 \%$ for the in-plane principal stresses. Therefore, in order to reduce the computational time, a uniform mesh size of $110 \mathrm{~mm}$ (resp. $20 \mathrm{~mm}$ ) has been chosen for the simulation of large (resp. intermediate) scale geometries.

The Tsai-Hill failure criterion is now considered to estimate the macroscopic strength of the Textile Module. According to the TsaiHill failure theory [15] the macromechanical failure criterion for anisotropic materials is given by

$I_{F}=\frac{\sigma_{L L}^{2}}{f_{m, L}^{2}}-\frac{\sigma_{L L} \sigma_{T T}}{f_{m, L}^{2}}+\frac{\sigma_{T T}^{2}}{f_{m, T}^{2}}+\frac{\sigma_{L T}^{2}}{f_{v}^{2}} \leqslant 1$

where $\sigma_{L L}, \sigma_{T T}$ and $\sigma_{L T}$ are the local stresses in the orthotropic material directions. In Eq. (3), $f_{m, L}, f_{m, T}$ and $f_{v}\left(=f_{v, L}=f_{v, T}\right)$ are bending and in-plane shear strengths of the laminated wood materials that
Table 2

Construction stresses values at point B for TM0, TM1and TM2.

\begin{tabular}{lccr}
\hline & TM0 & TM1 & \multicolumn{1}{c}{ TM2 } \\
\hline$\sigma_{L L}(\mathrm{MPa})$ & 45.1 & 18.01 & 9.61 \\
$\sigma_{T T}(\mathrm{MPa})$ & -3.3 & -0.302 & -0.61 \\
$\sigma_{L T}(\mathrm{MPa})$ & 0.65 & 0.248 & 0.48 \\
\hline
\end{tabular}

Table 3

$z$-Coordinate of points $1-5$ in global frame $(x, y, z)$.

\begin{tabular}{llllll}
\hline & Point 1 & Point 2 & Point 3 & Point 4 & Point 5 \\
\hline Experimental values $(\mathrm{mm})$ & 198 & 271 & 135 & 220 & 221 \\
FE values (mm) & 202 & 293.6 & 156.4 & 257 & 257.5 \\
\hline
\end{tabular}

are given in Table 1 . Values of $I_{F}$ greater than 1.0 may lead to failure. For each module, the Tsai-Hill criterion $I_{F}$ is visualized in Fig. 13b. Inspection of Fig. 13b, clearly indicates that maximum values of $I_{F}$ are located at the contact point B (the most critical point of the Textile Module during the assembly process). Table 2 gives the numerical values of the stress components at point $B$ for prototypes TM0, TM1 and TM2. According to the Tsai-Hill criterion, a module having the same characteristics as prototype TMO is predicted as likely to fail during the assembly process as was experimentally observed in the laboratory.

Although the application of this criterion also strictly predicts failure for TM1 (see Fig. 13b), it should be noted that the characteristic value of $f_{m, L}$ at fifth percentile $\left(f_{m, L}=15.8 \mathrm{MPa}\right)$ provided by the manufacturer for GFP material and used to calculate the TsaiHill criterion, is rather conservative. Three-point bending tests, carried out on a sample size of 26 GFP plates of size $486 \times 50 \mathrm{~mm}^{2}$, revealed that the median value of the bending resistance of GFP material is about $24 \mathrm{MPa}$. Nevertheless, due to the presence of
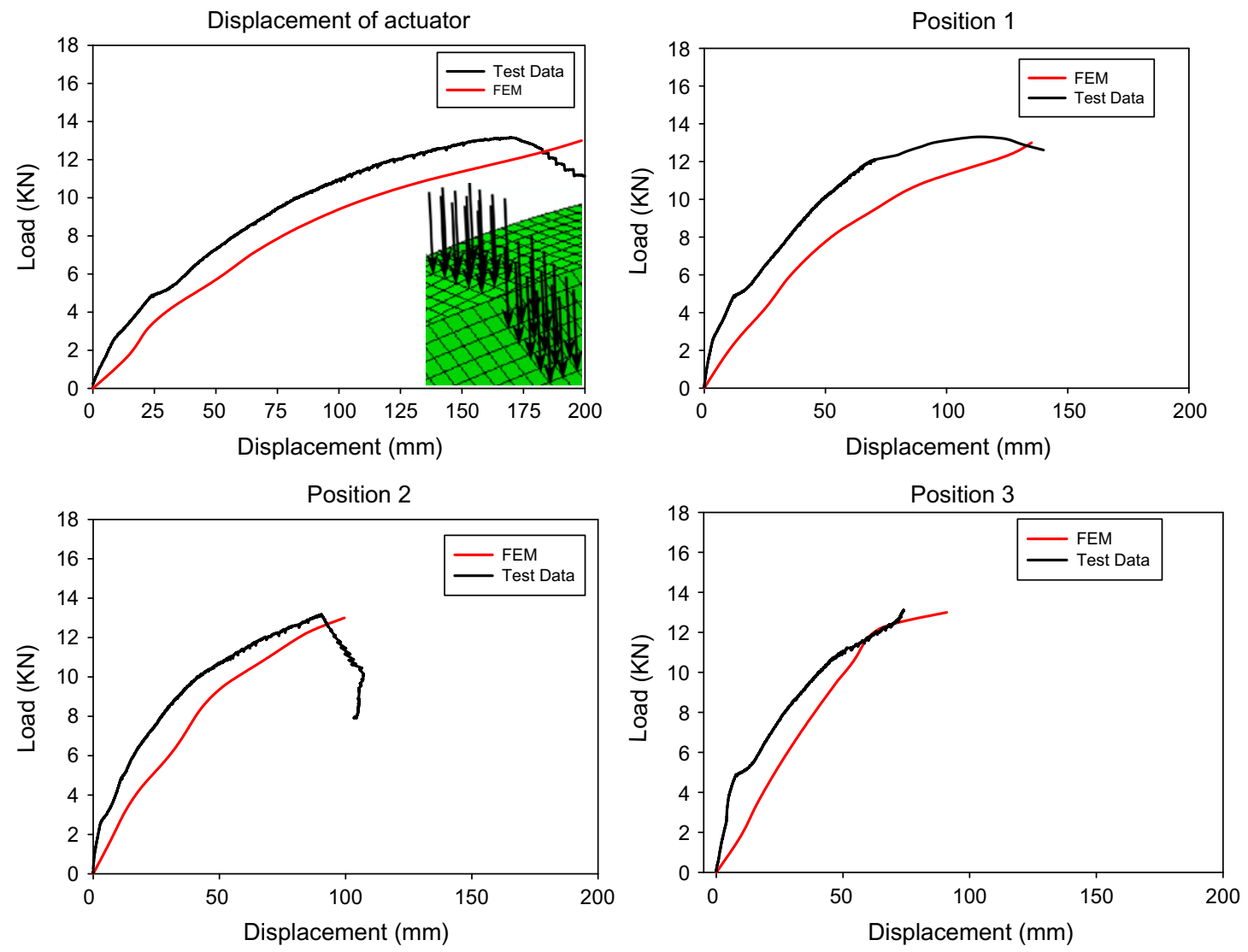

Fig. 14. Experimental and simulated force-displacement curves (TM1 prototype). 

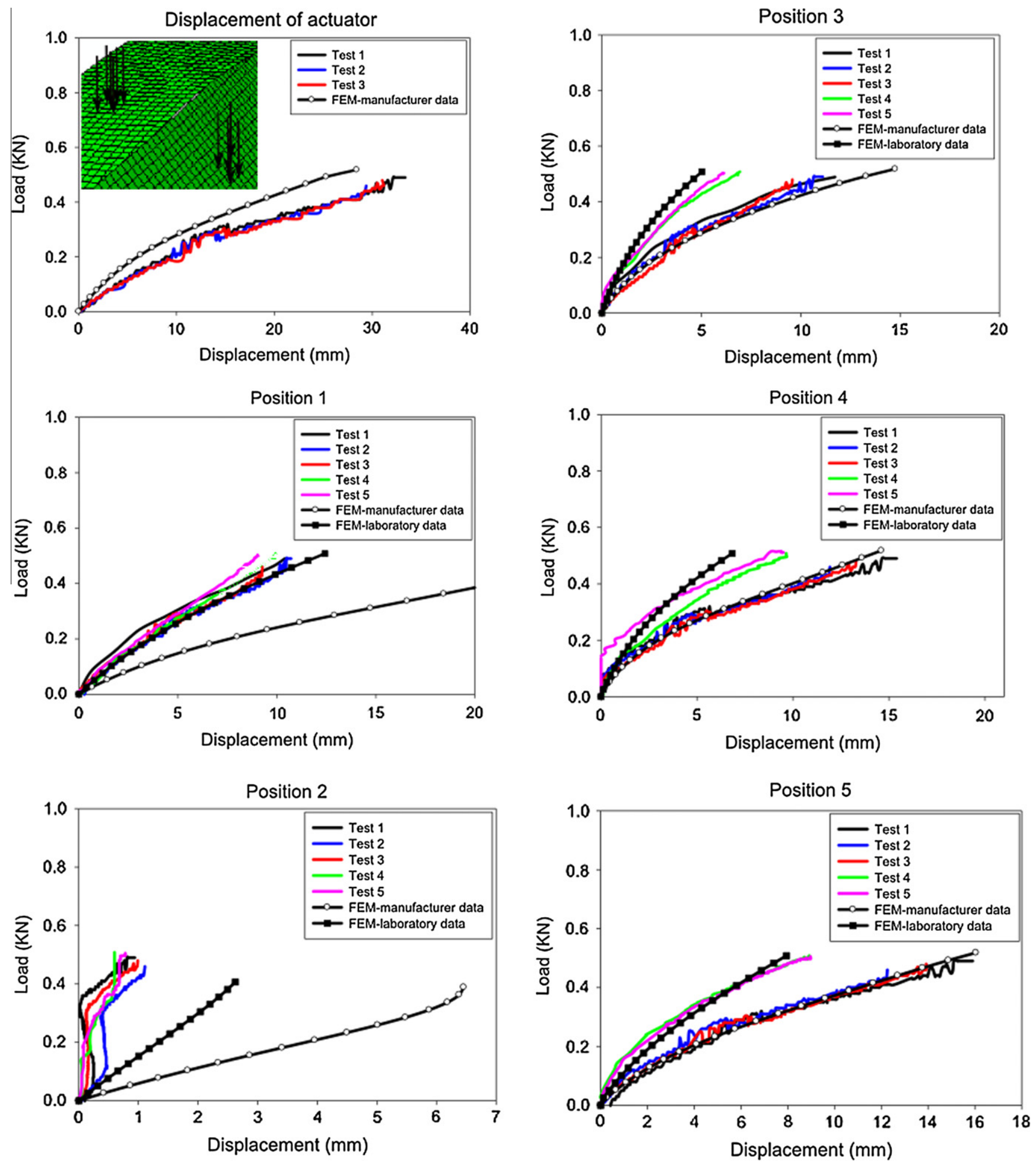

Fig. 15. Experimental and simulated force-displacement curves (TM2 prototype).

knots and other irregularities in the wood, a relatively large variability in the results was observed with bending strength values ranged from 4.3 to $55 \mathrm{MPa}$. If one takes the median value of $f_{m, L}$ $\left(f_{m, L}=24 \mathrm{MPa}\right)$, the maximum value of $I_{F}$ obtained for TMO is 5.58 while for TM1 the maximum value of $I_{F}$ obtained is 0.62 , a value in accord with our experimental observation that the TM1 module systematically withstands the forces applied during its construction.

\section{Results and discussion}

To compare the experimental and simulated initial shapes for TM2, the $z$-coordinates of points $1-5$ (indicated in Fig. 11b) in the global frame $(x, y, z)$ are reported in Table 3 . It can be seen that a good agreement exists between the values obtained from FE model and those measured with the prototype. The maximum discrepancy is found to be $14 \%$ at point 4 . 
Force-displacement curves are displayed in Fig. 14 for prototype TM1, where the vertical displacements are recorded at locations (points) 1, 2 and 3 (see Fig. 11a). Compressive loads are given here with positive values. The top left figure provides a plot of the recorded load versus actuator displacement. Experimental results indicate a nonlinear response of the structural element with a maximum sustained load of about $13 \mathrm{kN}$ that is reached when the actuator moves down to $170 \mathrm{~mm}$. At point 1 , this maximum load leads to a deflection of $120 \mathrm{~mm}$ for the Timber Module (top right figure). Smaller and comparable deflections of about $80 \mathrm{~mm}$ are obtained at points 2 and 3, farther from the loaded surface. Note that the value at point 1 is much more than the maximum deflection (span length $/ 300=33.5 \mathrm{~mm}$ ) of the serviceability limit state, indicating some inherent flexibility of the structure.

Numerically, the module is loaded at midspan such that half of the total (vertical) force is uniformly distributed over a small $\left(600 \times 300 \mathrm{~mm}^{2}\right)$ rectangular region of each panel (Fig. 14). In Abaqus, a second "loading" step has been added to the first building step (see Section 3.3), allowing the bending load to be applied on the simulated (prestressed) Textile Module. The applied load is linearly ramped over the step up to $13 \mathrm{kN}$. Simulated force-displacement curves are given in Fig. 14 at the corresponding experimental positions. The actuator displacement, obtained from the FE model, corresponds to the displacement of a node in the center of rectangular load area. The graphs indicate a nonlinear (elastic) response of the structure to bending load. The experimental decrease of the load (after $13 \mathrm{kN}$ ) is clearly not reproduced in the simplified elastic FE model considered.

It is observed that the numerical model tends to underestimate the rigidity of the structure. The discrepancy between the experimental and simulated curves might be primarily attributable to the material parameters used. Moreover, the assembly conditions considered for the large panels are clearly less controlled than those considered for the intermediate-scale specimen. The idealized "pinned connection" could not accurately represent the more complex (real) assembly conditions in this case. Nevertheless, relatively good agreement is observed between the experimental and numerical predictions.

Plotted in Fig. 15 are the force-displacement curves obtained for the prototype TM2, when loaded up to $0.5 \mathrm{kN}$. Compressive loads are again given with positive values. To verify good reproducibility, the loading procedure was repeated three times and the corresponding curves are referred to as Test1, Test2, Test3 in Fig. 15. A first series of tests was conducted just after receiving the Okoumé material from the manufacturers. A second series of tests (Test 4 and Test 5) was carried out on samples of the same batch of panels about eight months later, applying the same experimental procedure. The experimental results indicate an overall nonlinear response of the structure. Figures labeled "Position $i$ " $(i=1 \ldots 5)$ refer to vertical displacements obtained with transducers positioned at points $i(i=1 \ldots 5)$ as indicated in Fig. 11b. Interestingly, it appears that certain regions of the structure flex upward during the loading test. Specifically, we observed that all of the considered points shift down (towards the ground) except for point 1 which is shifted in the opposite direction (positive direction of $z$-axis). However for simplicity of graphic representation, all of the displacements have been plotted with positive values in the graphs.

Fig. 15 indicates, in particular at positions 3, 4 and 5, lower displacements for tests 4 and 5 than those obtained in the first series of tests with the same loading conditions. This effect might be attributable to a change in the environmental conditions. At $0.5 \mathrm{kN}$, a displacement amplitude of $10 \mathrm{~mm}$ is measured at point 1 that is comparable to the one recorded at the opposite point 3 (for the same panel). The vertical displacement at point 2 remains surprisingly small (less than $1 \mathrm{~mm}$ ) compared to the other.
Nevertheless, the curves of the second series of tests indicate the same trend even though the sudden increase of the slope observed for the first series after $0.5 \mathrm{~mm}$ no longer appears.

The largest displacements (i.e. about $15 \mathrm{~mm}$ ) are encountered at positions 4 and 5 . Potential wedge displacements can be recorded with transducers that are positioned horizontally at the two checking positions 6 and 7 (see Fig. 11b). A maximum (horizontal) displacement of $0.2 \mathrm{~mm}$ is recorded at these points for the wedges. This indicates a relatively small rotation of the wedge elements due to the bending load. Numerically, the wedges are fixed and the displacement is accordingly zero.

Depicted in Fig. 15 are the load-displacement curves obtained with the FE model by considering the manufacturer and laboratory material data. Half of the total (vertical) force is uniformly distributed over a small circular disk of radius $15 \mathrm{~mm}$ of each panel (Fig. 15). The applied load is ramped linearly over the step up to $0.5 \mathrm{kN}$. Using the manufacturers data (labeled "FEM-manufacturer data" in the legend), the numerical model correctly reproduces the experimental behavior of the Textile Module at positions 3, 4 and 5. The experimental trend is also satisfactorily recovered at position 1 . Introducing the elastic moduli from the laboratory measurements in the FE model ("FEM-laboratory data" in the legend), one recovers the experimental load-displacement curves of test 4 and 5 at positions 1,3 and 5 . It is worth noting that these material data were obtained during the period corresponding to the second series of tests and in the same environmental conditions. The model seems not to be able to reproduce the small displacements recorded experimentally at position 2 and significantly overestimates them in the load range considered.

The effects of tangential friction between the contacting panels have also been addressed, taking the values $\mu=0.3$ and $\mu=0.5$ for the coefficient of friction. The simulations indicate that these effects do not influence the global behavior of the structure in our case. They are not reported here while the plots are similar to those presented in Fig. 15. It is concluded that no sliding occurs between the surfaces of the panels.

Finally, the introduction of wedge elements significantly affects the overall rigidity of the structure. Considering the TM2 geometry once again, Fig. 16 compares the simulated displacement-load curves obtained at points 3 and 4 when the structure is assembled with pin and wedge connections. In both cases, the same material (i.e. Okoumé plywood) is considered and the modules are loaded up to $0.5 \mathrm{kN}$. As expected, the wedge connecting elements provide more rigidity for the structure than pin connections.

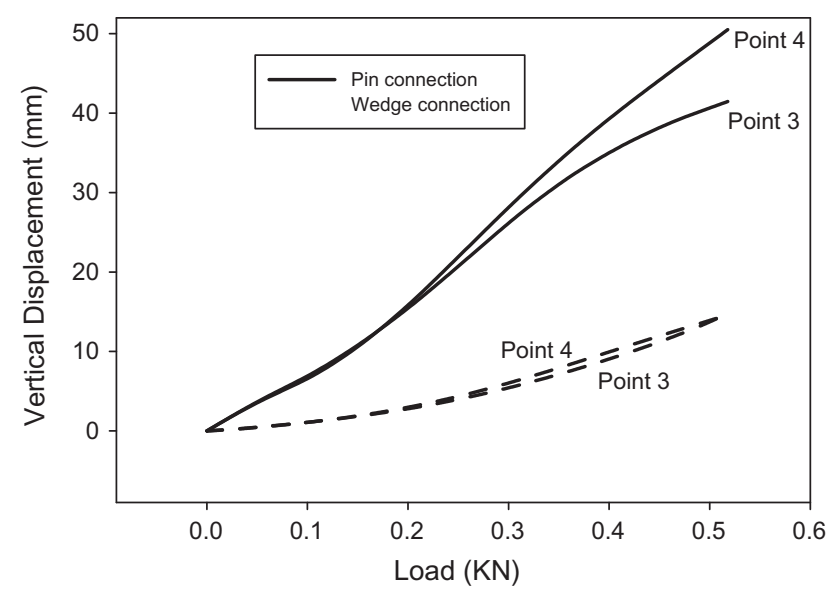

Fig. 16. Simulated displacements at points 3 and 4 for pin and wedge connections (TM2 geometry). 


\section{Conclusion}

In this work, a novel class of timber structures based on the logic and principles of textile techniques has been investigated. A geometrically nonlinear finite element model has been developed for the construction of a single Textile Module including pinned and so-called wedge connections for the assembly conditions. For comparison, large-scale and intermediate-scale experimental prototypes with the previous connections have been constructed.

The proposed analysis aimed first at reproducing the initial shape of the structure and thus evaluating the resulting construction (initial) stresses induced during the assembly process. It was shown that the simulated shape could satisfactory fit to the experimental one at several measurement points. Moreover, the anisotropic Tsai-Hill criterion based on the maximum induced stresses allows one to select safe design parameters. It was observed that a length-to-width ratio $l / w=7.5$ for the large-scale GFP panels leaded to failure during the construction while $l / w=15$ was safe. For the intermediate prototype $(l / w=9.75)$, wedge connections comparatively lead to lower levels of construction stresses and could be adopted at larger scale.

Secondly, the structural behavior of the Textile Module has been examined under bending tests. For the two considered geometries, the resulting deflections have been measured and calculated at several locations. They highlight a nonlinear bending response of the Textile Module. A good agreement is generally observed between the experimental results and the FE predictions at intermediate and large scales. Finally, the introduction of wedge elements was found to significantly improve the overall rigidity of the Textile Module.

\section{Acknowledgements}

The authors would like to thank the Swiss National Science Foundation for supporting this work through Contract
No. 200021-126802. They are also grateful to Mrs. Maria Lindqvist for her participation in the experimental tests.

\section{References}

[1] Langenbach R. Resisting earth's forces: typologies of timber buildings in history. Struct Eng Int: J Int Assoc Bridge Struct Eng (IABSE) 2008;18:137-40.

[2] König H. Bauen mit Holz als aktiver Klimaschutz. Bauen mit Holz: Wege in die Zukunft. München: Prestel Verlag; 2011.

[3] Iffland JSB. Folded plate structures. ASCE J Struct Div 1979;105:111-23.

[4] Happold E, Liddell WI. Timber lattice roof for the Mannheim Bundesgartenschau. Struct Eng 1975;53:99-135.

[5] Kelly OJ, Harris RJL, Dickson MGT, Rowe JA. Construction of the downland gridshell. Struct Eng 2001;79:25-33.

[6] Douthe C, Baverel O. Design of nexorades or reciprocal frame systems with the dynamic relaxation method. Comput Struct 2009;87:1296-307.

[7] Popovic Larsen O. Reciprocal frame architecture. London: Architectural Press; 2007.

[8] Weinand Y. Innovative timber constructions. J Int Assoc Shell Spatial Struct 2009;50:111-20.

[9] Weinand Y, Hudert M. Timberfabric: applying textile principles on a building scale. Architect Des 2010;80:102-7.

[10] D'Amato E. Finite element modeling of textile composites. Compos Struct 2001;54:467-75

[11] D'Amato E. Nonlinearities in mechanical behavior of textile composites Compos Struct 2005;71:61-7.

[12] Kalidindi SR, Franco E. Numerical evaluation of isostrain and weightedaverage models for elastic moduli of three-dimensional composites. Compos Sci Technol 1997;57:293-305.

[13] Page J, Wang J. Prediction of shear force using 3D non-linear FEM analyses for a plain weave carbon fabric in a bias extension state. Finite Elem Anal Des 2002;38:755-64.

[14] Whitcomb J, Woo K, Gundapaneni S. Macro finite element for analysis of textile composites. J Compos Mater 1994;28:607-18.

[15] Daniel I, Ishai O. Engineering mechanics of composite materials. Oxford, UK: Oxford University Press; 1994.

[16] Bodig J, Jayne BA. Mechanics of Wood and wood composites. Van Nostrand Reinhold Company; 1982.

[17] Abaqus: abaqus theory manual version 6.9; 2009

[18] Ertas A, Krafcik JT, Ekwaro-Osire S. Performance of an anisotropic Allman/DKT 3-node thin triangular flat shell element. Compos Eng 1992;2:269-80.

[19] Murthy SS, Gallagher RH. A triangular thin-shell finite element based on discrete Kirchhoff theory. Comput Methods Appl Mech Eng 1986;54:197-222.

[20] Reddy JN. An introduction to non-linear finite element analysis. New York: Oxford University Press; 2004. 